\title{
La charte de Richer de Liège du 16 novembre 932. Une copie
} historique à réhabiliter

Florence Close

\section{Citer ce document / Cite this document :}

Close Florence. La charte de Richer de Liège du 16 novembre 932. Une copie historique à réhabiliter. In: Bulletin de la Commission royale d'histoire. Académie royale de Belgique. Tome 177, 2011. pp. 5-20;

doi : https://doi.org/10.3406/bcrh.2011.1108

https://www.persee.fr/doc/bcrh_0001-415x_2011_num_177_1_1108

Fichier pdf généré le 04/05/2018 


\title{
Résumé
}

Le 16 novembre 932, l'évêque de Liège Richer consacra un autel à la sainte Trinité en la cathédrale Saint-Lambert de Liège et le dota richement ; il consigna cette décision par écrit. La charte de fondation est parvenue jusqu'à nous sous la forme d'une copie historique isolée insérée dans les Gesta episcoporum Tungrensium et Leodiensium du chanoine Anselme de Liège. Cet article entend souligner l'intérêt de ce document tant pour l'histoire de la chancellerie liégeoise que pour celle de la réforme religieuse carolingienne dans l'espace lotharingien. II comporte en annexe une nouvelle édition critique de ce document établie sur la base d'une confrontation de sept copies réparties en deux classes selon une typologie différente de celle de R. Koepke, l'éditeur de la chronique d'Anselme pour les Monumenta Germaniae Historica.

Op 16 november 932 wijdde de Luikse bisschop Richer een altaar aan de Heilige Drievuldigheid in de Sint-Lambertuskathedraal van Luik dat hij rijkelijk begiftigde. Hij stelde deze beslissing te boek. Het stichtingscharter is tot vandaag overgeleverd in de vorm van een geïsoleerde historische kopie die is opgenomen in de Gesta episcoporum Tungrensium et Leodiensium van de Luikse kanunnik Anselmus. In dit artikel wil de auteur het belang van dit document onderstrepen, zowel voor de geschiedenis van de Luikse kanselarij als voor de geschiedenis van de Karolingische religieuze hervorming in Lotharingen. Het artikel heeft als bijlage een nieuwe kritische editie van dit document, opgesteld op basis van een confrontatie van zeven kopieën die in twee klassen zijn verdeeld volgens een typologie die verschilt van die van R. Koepke, uitgever van de kroniek van Anselmus voor de Monumenta Germaniae Historica.

\begin{abstract}
On 16 November 932, Richer, bishop of Liège, devoted an altar to the Saint Trinity in Liège's St. Lambert's Cathedral and lavishly decorated it, a decision he recorded in writing. We obtained the foundation charter as an isolated historical copy, inserted into the Gesta episcoporum Tungrensium et Leodiensium from the Anselm of Liège canon. The article stresses the interest of this document as much for the history of the chancellery of Liège as for the Carolingian religious reform in the Lotharingian space. It has as an annex a new critical edition of the document, established based on a confrontation of seven copies divided into two classes according to a typology different from that of $\mathrm{R}$. Koepke, editor of the Anselm chronicle for the Monumenta Germaniae Historica.
\end{abstract}




\title{
La charte de Richer de Liège du 16 novembre 932 Une copie historique à réhabiliter*
}

\author{
par Florence Close \\ FNRS Liège
}

Le 16 novembre 932, l'évêque Richer de Liège consacra un autel à la sainte Trinité dans la cathédrale Saint-Lambert de Liège et le dota richement ; il consigna cette décision par écrit ${ }^{1}$. Ce document diplomatique est assurément l'un des plus célèbres de l'histoire du diocèse de Liège au haut Moyen Âge. À divers titres, il a su s'imposer comme un témoin primordial. Il est, tout d'abord, l'un des plus anciens actes épiscopaux liégeois connus ${ }^{2}$. Par ailleurs, il contient sinon la plus ancienne, du moins l'une des premières attestations de dédicace d'un autel à Dieu trine dans une église séculière. En cela, il constitue, par son objet, un intéressant jalon de l'histoire du culte trinitaire dans l'Église latine occidentale. Enfin, Richer y affirme que son prédécesseur Étienne (901-920) composa un office liturgique en l'honneur de la Trinité or, au vu de récentes conclusions, celui-ci semble correspondre à l'office qui fut chanté dans l'Église catholique romaine jusqu'au concile

* Cet article a bénéficié des conseils des professeurs J.M. Duvosquel, J.L. Kupper, L. Milis et R. Van Caenegem, membres du comité de lecture du BulleTIN DE LA COMMISSION ROYALE D'HistoIRE (ci-dessous: $B C R H$ ); qu'ils trouvent ici l'expression de notre reconnaissance. L'édition critique en annexe n'aurait pu être établie aussi aisément sans la précieuse collaboration des responsables de la conservation et de la reproduction des manuscrits étudiés: mesdames A. Ilginus (Herzog August Bibliothek Wolfenbüttel) et M.-P. Laffitte (Bibliothèque Nationale de France), le père Herman Janssens (Abdij der Norbertijnen van Averbode), monsieur Olivier Donneau (Bibliothèque de l'Université de Liège). Qu'ils soient assurés de notre gratitude.

1 A. Wauters, St. Bormans, J. Halkin et al., Table chronologique des chartes et diplômes imprimés concernant l'histoire de la Belgique, t. 1, Bruxelles, 1866, p. 342. - Assez étonnamment, cette charte a été écartée du corpus diplomatique rassemblé dans le Thesaurus Diplomaticus, [CDRom], Turnhout, Brepols, 1997.

${ }^{2}$ La plus ancienne charte épiscopale liégeoise conservée est un fragment d'un acte de l'évêque Walcaud délivré vers 815. Le premier original vrai est une lettre du 19 juin 980 adressée par l'évêque Notger à l'abbaye de Saint-Bavon de Gand (J.-L. KuPPER, La "chancellerie" des évêques de Liège. $x^{*}-x_{I} f^{2}$ siècles, à paraître dans les actes du colloque Chancelleries princières et Scriptoria dans les anciens Pays-Bas $x^{e}-X V^{2}$ siècles / Vorstelijke kanselarijen en Scriptoria in de Lage Landen 10de-15de eeuw tenu au Palais des Académies de Bruxelles les 11-12 décembre/december 2009 à l'occasion du $175^{\mathrm{e}}$ anniversaire de la Commission Royale d'Histoire, $B C R H(2010)$, n. 9. 
de Vatican II $^{3}$. Le contenu de cet acte constitue, par conséquent, l'un des principaux arguments en faveur de l'origine liégeoise de la fête liturgique de la Trinité 4 . Davantage encore que son contenu, l'histoire de la tradition de ce texte semble avoir contribué à accroître sa notoriété car nul ne connaît la forme originale ni la teneur intégrale de ce document. La charte de l'évêque Richer de Liège (921-945) du 16 novembre 932 est, en outre, un exceptionnel exemple de copie dite «historique». Elle n'est parvenue jusqu'à nous que sous la forme d'une copie libre isolée, insérée dans une source narrative: les Gesta episcoporum Tungrensium, Traiectensium et Leodiensium du chanoine Anselme de Liège. À en croire l'auteur de cette chronique, cet acte pourrait avoir été copié sinon directement sur l'original, du moins sur une copie proche de la source. Le chanoine liégeois découvrit, en effet, vraisemblablement l'exemplar - le modèle, l'original, l'archétype - dans les archives de la cathédrale de Liège (armarium $)^{5}$. Il en copia l'essentiel de la teneur dans le vingt-et-unième paragraphe de ses Gesta episcoporum. En l'absence d'autres témoins manuscrits, l'histoire et la tradition de la charte du 16 novembre 932 demeurent intrinsèquement liées à celles de la chronique d'Anselme du XI ${ }^{\mathrm{e}}$ siècle.

\section{La charte de 932 dans la tradition manuscrite de la chronique d'Anselme de Liège}

La chronique d'Anselme est toujours dans l'attente d'une bonne édition critique $^{6}$. Le meilleur texte dont nous disposions à l'heure actuelle est celui qui fut édité par R. Koepke pour les Monumenta Germaniae Histo-

3 A. Auda, L'école musicale liégeoise au x siècle. Étienne de Liège, Bruxelles, 1923; Fl. Close, L'office de la Trinité d'Étienne de Liège (901-920). Un témoin de l'héritage liturgique et théologique de la première réforme carolingienne à l'aube du $x^{e}$ siècle, REVUE BELGE de Philologie ET D'Histoire, t. 86 (2008/4), pp. 623-643.

${ }^{4} \mathrm{Fl}$. Close, Le rôle de l'Église de Liège dans le développement du culte de la Trinité en Europe, mémoire de licence inédit, Université de Liège, année académique 1999-2000; EAD, L'octave de la Trinité du calendrier liégeois. Chronologie et liturgie, LE MOYEN ÂGE, t. 110/3-4 (2004), pp. 627-641.

5 ANSELME DE LIÈGE, Gesta episcoporum Tungrensium, Traiectensium et Leodiensium, $\S 20$, éd. R. KoEpKe, $M G H S S$, t. 7, Hanovre, 1846, p. 200, 1. 21-22. - Sur la signification à donner à ces termes latins: J.-Fr. NIERMEYER, C. VAN DE KIEFT, Mediae latinitatis Lexicon Minus, Leyde, 1976, sv. Exemplar et sv. Armarium; A. BlAISE, Dictionnaire latin - français des auteurs du moyen âge, Turnhout, Brepols, 1975, sv. Armarium.

${ }^{6}$ Sur ce souhait largement partagé de voir paraître une nouvelle édition de cette chronique, voir les commentaires glissés dans les plus récents répertoires bibliographiques: Repertorium Fontium Historiae Medii Aevi, t. 2, Rome, 1967, pp. 367-368; Narrative Sources. The narrative Sources from the medieval Low Countries, [en ligne], http://www.narrative-sources.be/ sv. Anselmus leodiensis [mise en ligne 2.12.2010]; Geschichtsquellen des deutschen Mittelalters, t. 1 (A), Bayerische Akademie Der Wissenschaften, novembre 2009, pp. 153-154 [ligne] http://www.repfont.badw.de/A.pdf. 
rica en $1846^{7}$. Or, elle fut très tôt contestée. Le premier à avoir porté un sévère regard critique sur l'édition de cette chronique fut l'éminent médiéviste liégeois G. Kurth ${ }^{8}$. Il reprocha principalement à l'éditeur allemand d'avoir écarté les manuscrits liégeois rangés dans la classe $B$ pour ne baser son travail que sur les trois manuscrits et la copie imprimée qu'il regroupa dans la classe $A^{9}$. G. Kurth attira alors l'attention sur une copie inconnue de R. Koepke: un manuscrit du Xvi ${ }^{e}$ siècle conservé dans les archives de l'abbaye d'Averbode ${ }^{10}$. Le médiéviste liégeois démontra avec beaucoup de conviction que cette copie, quoique tardive, pourrait avoir été copiée directement sur le manuscrit original. Il contribua ainsi par ses recherches à la réhabilitation de deux manuscrits liégeois autrefois disséminés dans l'espace européen et désormais conservés à la salle des manuscrits de l'Université de Liège. Le plus ancien est une copie du XIII ${ }^{\mathrm{e}}$ siècle provenant de l'abbaye d'Aulne; celle-ci était déjà mentionnée dans l'édition de Chapeaville au début du $\mathrm{XVII}^{\mathrm{e}}$ siècle ${ }^{11}$. Le deuxième fut copié sur papier en 1606 par le chanoine de saint Materne de la cathédrale Saint-Lambert de Liège, Daniel Raymundi, au départ d'un manuscrit intitulé De gestis episcoporum Leodiensis Breviarium daté, selon le copiste, de $1056^{12}$. Il fut conservé à la collégiale Saint-Martin de Liège puis dans la bibliothèque de $M$. de Theux avant de rejoindre les collections universitaires ${ }^{13}$. Une nouvelle édition de la chronique d'Anselme exigerait la prise en compte de ces sept manuscrits, auxquels pourraient occasionnellement s'ajouter les leçons des copies abrégées et interpolées de ces gesta formant la classe C de la typologie de R. Koepke. Pour l'édition de la charte du 16 novembre 932, les sept premiers manuscrits suffiront; les versions interpolées et abrégées de la chronique ne contiennent qu'un résumé paraphrasé de la teneur de l'acte. Selon S. Balau, les deux manuscrits liégeois - Liège, BU, ms. 3173 (Aulne, $\mathrm{XIII}^{\mathrm{e}}$ siècle) et Liège, BU, ms. 1964 (XVII $^{\mathrm{e}}$ siècle) -

7 ANSELME DE LiÈGE, Gesta episcoporum Tungrensium, Traiectensium et Leodiensium, éd. R. KoEPKe, MGH SS., t. 7, Hanovre, 1846, pp. 189-234; pour la charte idem, $\$ 21$, pp. 200-201.

${ }^{8} \mathrm{G}$. KuRTh, Notice sur un manuscrit d'Hariger et d'Anselme conservé à l'abbaye d'A verbode, $B C R H, 4^{\text {e }}$ série, t. 2 (1875), pp. 377-394.

9 Sur le classement des manuscrits par R. Koepke, cf. $M G H S S$., t. 7, pp. 157-159- Pour les références de ces différents manuscrits, $\mathrm{cf}$. annexe.

10 Averbode, AA lezen Sectie IV, Nr. 12, pp. 66-67 ( $\mathrm{XvI}^{\mathrm{e}}$ siècle).

11 Liège, BU, ms. 3173 - Olim Cheltenham, Phillipps 435.

12 Jean Chapeaville (1551-1617) et ses amis. Contribution à l'historiographie liégeoise, édition critique du texte latin, traduction française et notes philologiques de René Hoven, introduction et annotations historiques de Jacques Stiennon, description bibliographique détaillée et notes complémentaires de Pierre-Marie Gason, Bruxelles, Académie royale, 2004 , p. 85 , n. 86.

13 Liège, BU, ms. 1964. - La bibliothèque universitaire de Liège conserve également une

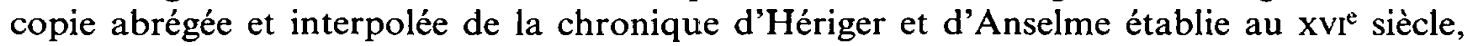
rangée par $R$. Koepke, avec les autres copies de ce type, dans la classe C (Liège, BU, ms. 178). C'est la version abrégée du diplôme de 932 contenue dans ce manuscrit qu'édita J. Chapeaville. (J. Chapeaville, Qui gesta pontificum Tungrensium, Traiectensium et Leodiensium scripserunt..., t. 1, Liège, 1612, pp. 170-171). 
comporteraient, malgré quelques erreurs de distraction du copiste, des leçons très proches sinon identiques à celles du manuscrit d'Averbode ${ }^{14}$. Cette affirmation mériterait d'être vérifiée et nuancée. En effet, la copie du $\mathrm{XVII}^{\mathrm{e}}$ siècle (Liège, BU, ms. 1964, fol. 91v-92r) propose des leçons épurées de bons nombres de déformations orthographiques propres au latin médiéval, très proches de celles d'Averbode. Par contre, en ce qui concerne le paragraphe 21 de la chronique d'Anselme, il ressort de notre travail de comparaison des diverses copies que le manuscrit d'Aulne autrefois rangé par $\mathbf{R}$. Koepke dans la classe $B$ - présente davantage de leçons communes aux manuscrits de la classe $A^{15}$. Il convient également de noter que, contrairement à ce que pensait $R$. Koepke, il semble peut probable que E. Martène et $U$. Durand aient édité la chronique d'Anselme sur la base du manuscrit de La Haye. Cette hypothèse ne se vérifie, en tous cas, pas dans l'étude comparative des copies historiques de l'acte qui retient notre attention; la copie imprimée du $\mathrm{XVIII}^{\mathrm{e}}$ siècle présente d'importantes variantes ainsi que le montrera notre nouvelle proposition d'édition critique de l'acte en annexe à cet article.

\section{Teneur de l'acte}

En l'absence d'une invocation divine, c'est une adresse à très forte connotation trinitaire qui inaugure le protocole initial de la charte. Cette première formule est intéressante à plus d'un titre. Tout d'abord, le destinataire de l'acte n'est pas, ainsi que l'on pourrait s'y attendre, le peuple des fidèles liégeois, les ouailles de Richer, mais «tous ceux qui confessent la Trinité » sans distinction d'appartenance à une Église régionale, à un diocèse. Richer souhaitait-il faire connaître sa décision au plus grand nombre ou était-ce alors la formule diplomatique en usage à Liège? La question mérite d'être posée; ces mots ne suffisent, en effet, pas pour affirmer que cette charte fut largement diffusée. L'histoire singulière de la tradition de ce document atteste d'ailleurs le contraire. On pourrait même s'interroger sur la promulgation effective de ce document en dehors de la cathédrale Saint-Lambert. Dans la suscription, Richer se dit évêque de l'Église de Tongres. Par ce titre, qui fait référence au plus ancien siège du diocèse mosan, le prélat insiste sur l'ancienneté de son diocèse ce qui est de nature à lui conférer une certaine dignité ${ }^{16}$. Cet acte passe pour le

14 S. BALAU, Les sources de l'histoire de Liège au moyen âge. Étude critique, Bruxelles, 1903, p. 132.

$15 \mathrm{Cf}$. annexe.

16 Étienne de Liège (901-920), le prédécesseur de Richer, l'utilisait également. (ÉTIENNE DE Liège, Lettre dédicace du liber capitularis, éd. C. Mohlberg, dans C. Mohlberg, Spuren eines verlorenen Liturgiebuches, der Liber capitularis Stephans von Tongern ( $\uparrow 920)$, dans A. Cauchies (dir.), Mélanges d'histoire offerts à Charles Moeller à l'occasion de son jubilé de 50 années de professorat à l'Université de Louvain (1863-1913), t. 1, Louvain, 1914, p. 357; 
dernier de l'histoire de la diplomatique épiscopale liégeoise à présenter cette titulature ${ }^{17}$. Cette formulation simple sans autre marque de dévotion s'explique par la longueur de l'adresse au propos théologique développé. Le protocole initial se clôt sur une formule de souhait de grâce et de paix en lieu et place du traditionnel salut. Toute cette première partie témoigne d'une indéniable dévotion à la Trinité.

Dans un préambule assez bref, Richer exprime son souci de mener à bien sa mission pastorale en signifiant au peuple chrétien ce qui est bon pour lui. Au terme d'une formule de notification sans intérêt particulier, il rappelle que l'office liturgique de la Trinité fut composé par son prédécesseur, Étienne, afin d'offrir à son Église le formulaire liturgique nécessaire pour rendre un culte à Dieu un et trine. Par cet exposé, l'évêque de Liège inscrit dans la continuité de l'œuvre de son devancier sa décision d'ériger un oratoire dans la cathédrale Saint-Lambert et d'y consacrer un autel dédié à la sainte Trinité. Le dispositif n'a pas été intégralement transcrit par Anselme. Seule la partie concernant la fondation de l'oratoire et de l'autel a été copiée. Le chanoine liégeois a résumé la suite du texte qui consistait, à ce qu'il semble, en une liste d'indications pratiques relatives aux luminaires et au service divin lors de la solennité. Cette partie centrale de l'acte comporte deux importantes digressions théologiques destinées à définir et qualifier la Trinité divine; elles sont également de la plume d'Alcuin.

$\mathrm{Si}$, dans sa transcription, le chanoine Anselme a amputé l'acte des dispositions relatives à la dotation de l'autel ${ }^{18}$, il a, en revanche, fidèlement reproduit la formule de datation, ce qui nous permet d'affirmer que la charte fut délivrée le 16 des calendes de décembre soit le 16 novembre 932.

\footnotetext{
ÉTIENNE DE LIĖGE, Vita sancti Landiberti episcopi traiectensis, éd. B. KRUSCH, MGH SRM, t. 6, Hanovre-Leipzig, 1913, p. 385.) Faisant nôtre une hypothèse de Guy Philippart, nous avons suggéré en d'autres lieux qu'Étienne de Liège a lutté pour réhabiliter la réputation du diocèse mosan (G. PhilipPart, Hagiographies Locale, Régionale, Diocésaine, Universelle. Les hagiographies du saint patron dans l'aire belge du x" siècle, dans W. BERSCHIN (éd.), Lateinische Kultur im X. Jahrhundert, Akten des I. Internationalen Mittellateinerkongresses. Heidelberg, 12-15.IX.1988, Stuttgart, Anton Hiersemann, 1991 (Mittellateinisches Jahrbuch, t. 24-25 (1989-1990), p. 362; Fl. Close, L'office de la Trinité. Liège, Metz, Mayence, Saint-Gall, à paraître dans lcs actes du colloque international Notger et son temps organisé à Liège du 19 au 21 novembre 2008, éd. A. Wilkin, J.L. KuPPER, A. Dierkens. - Sur Notger et son œuvre: J.P. Del Ville, J.L. KuPPer, M. LAFfineur-CréPIN (dir.), Notger et Liège. L'an mil au caur de l'Europe, Liège, Perron, 2008.

17 J.L. KuPPER, Leodium (Liège/Luik). Historia, dans S. WeinfurTer, O. Engels (éd.), Series episcoporum ecclesiae catholicae occidentalis ab initio usque ad annum MCXCVIII, Series V, Germania, t. 1 Archiepiscopatus Coloniensis, Stuttgart, Hiersemann, 1982, p. 44, n. 3.

18 Sequitur deinceps cuiusdam fundi ad idem altare traditio, unde ut ibidem continentur luminaria et reliqua divinae servitutis iugiter adimpleantur solemnia quae hic interponere supervacuum extimavi. (éd. R. KOEPKE, MGH SS, t. 7, pp. 200-201, 1. 44-2.
} 


\section{La charte du 16 novembre 932 dans la chronique d'Anselme}

En décidant, entre 1052 et 1056, de continuer les Gestes des évêques de Liège rédigées par Hériger de Lobbes $(\dagger 1007)$, Anselme de Liège n'avait d'autre but que celui de célébrer le glorieux passé intellectuel du diocèse de Liège à la tête duquel l'évêque Wazon (1042-1048) avait été placé ${ }^{19}$. Il souhaitait mettre en exergue les hauts faits des prélats du diocèse mosan, établir une "lignée sainte», au sein de laquelle Wazon apparaîtrait "comme l'héritier de toutes les forces spirituelles, morales et intellectuelles capitalisées par ses devanciers ${ }^{20}$. Si Anselme décida de recopier partiellement la charte du 16 novembre 932, c'est que celle-ci devait servir les intérêts de son œuvre. Nous pouvons, par conséquent, supposer qu'il limita son emprunt aux passages contenant les informations nécessaires et suffisantes à son propos, en l'occurrence l'exposé et la première partie du dispositif. Il faut alors, d'une part, remarquer que, dans le paragraphe qui précède immédiatement la transcription de l'acte, Anselme affirme qu'on croyait de son temps que l'office de la Trinité avait été composé par Hucbald de Saint-Amand ${ }^{21}$ et, d'autre part, accorder toute l'attention nécessaire à l'intitulé du paragraphe reprenant la copie de l'acte: exemplar testamenti in quo testatur Richarius Stephanum condidisse responsoria de s. Trinitate ${ }^{22}$. Ces deux indices nous permettent d'émettre l'hypothèse que l'attribution de la composition de cet office à Etienne de Liège devait, dans l'idée du chanoine liégeois, apporter au diocèse mosan quelques lettres de noblesse ${ }^{23}$. La copie de la charte épiscopale de Richer pourrait ainsi avoir répondu à deux impératifs majeurs: tout d'abord rétablir une vérité historique mise à mal dans le diocèse $a u \mathrm{xI}^{\mathrm{e}}$ siècle, en l'occurrence la composition de l'office de la Trinité par un évêque liégeois; ensuite, inscrire la fondation de l'autel de la Trinité de la cathédrale de Liège, dans la continuité de l'œuvre liturgique d'Étienne, en vue de donner naissance à un culte rendu à Dieu trine.

En composant son office, Étienne de Liège avait offert aux communautés chrétiennes qui le souhaitaient la possibilité de consacrer un jour complet au culte de la Trinité. L'érection de l'autel par Richer, son succes-

19 S. BALAU, Les sources de l'histoire de Liège, pp. 131-132 et 162-172; J.-L. KuPPER, Les Gesta pontificum Leodicensis aecclesiae du chanoine Anselme, dans Problématique de l'histoire liégeoise, Liège, 1981, pp. 29-41.

20 J.-L. KuPPER, Les Gesta pontificum Leodicensis, p. 36.

21 Idem ipse responsoriorum, quae de sancta Trinitate cantantur, auctor fuit, quod nos quidem ignorabamus, credentes, quod fama habebat, nescio quem Hubaldum ipsorum fuisse compositorem. (ANSELME, Gesta episcoporum, § 20, p. 200, 1. 19-21). - Sur l'œuvre d'Hucbald de Saint-Amand: Y. Chartier, Clavis operum Hucbaldi Elnonensis: Bibliographie des auvres d'Hucbald de Saint-Amand, dans The Journal of Medieval Latin, t. 5 (1995), pp. 202-224.

22 Cf. annexe.

23 Fl. Close, «L'office de la Trinité. Liège, Metz, Mayence, Saint-Gall». 
seur, avait probablement rehaussé le prestige de cette solennité en offrant aux Liégeois un lieu privilégié pour la célébrer. L'adresse universelle de l'acte pourrait être envisagée comme un indice d'une invitation à promouvoir ce culte, lancée aux Églises voisines. On remarquera, toutefois, dans ce document, la trace de la conscience qu'avait Richer des dérives possibles de l'instauration d'une telle fête: l'évêque estimait primordial que cette insertion dans le calendrier ne contribue pas à réserver les louanges dues à Dieu dans sa Trinité à un jour et à un lieu précis. Ce n'est pas sans raison qu'il rappela solennellement la place due au culte de la Trinité dans toutes les assemblées eucharistiques ${ }^{24}$. Par ces mots, Richer soulignait déjà les arguments qui seraient avancés par Rome au siècle suivant pour justifier le refus pontifical d'instituer cette fête dans l'Église universelle ${ }^{25}$. La retranscription de la charte du 16 novembre 932 dans la chronique liégeoise rédigée au milieu du $\mathrm{XI}^{\mathrm{e}}$ siècle pourrait, par conséquent, avoir été motivée par un autre débat de bien plus grande ampleur: celui de l'opportunité d'institutionnaliser la fête de la Trinité. Les premières oppositions romaines à cette fête coïncident, en effet, avec l'époque de rédaction des Gesta episcoporum. Les textes n'attestent pas la prise de position du pape contre cette solennité avant le pontificat d'Alexandre II (1061-1073) mais rien n'empêche de supposer que quelques voix se fussent déjà élevées, quelques années auparavant, pour encourager l'évêque de Rome à se prononcer sur cette question ${ }^{26}$. Tandis que certaines Églises diocésaines dont celle de Rome - refusaient de consacrer un jour particulier au culte d'un dogme fondamental de la religion catholique, Anselme dut trouver dans cette charte l'expression du zèle avec lequel Richer avait exercé sa fonction pastorale. La charte du 16 novembre 932 suffisait à justifier le choix des Liégeois d'adopter une telle fête et de consacrer un jour particulier à la sainte Trinité dans le calendrier liturgique sans renoncer à la dévotion quotidienne due à Dieu.

En décidant d'insérer cet acte épiscopal dans sa chronique, Anselme souhaitait vraisemblablement affirmer le caractère ancestral de la haute culture, de la parfaite orthodoxie et de la profonde piété des évêques de Liège. Très probablement, entendait-il faire remonter bien au-delà de Notger (997-1008), premier prince-évêque de Liège et promoteur de

24 ...Quamvis a cunctis fidelibus Deus et Dominus vera et sempiterna unitas in substantia ubique fideliter adoretur, quia una substantia est Pater et Filius et Spiritus sanctus (ANSELME, Gesta episcoporum, $\$ 21$, éd. R. KOEPKE, p. 200, 1. 41-43).

25 Ecclesie siquidem Romana in usu non habet quod in aliquo tempore huiusmodi celebret specialiter festivitatem quum singulis diebus "gloria Patri et Filio et Spiritu sancto" et cetera similia dicantur ad laudem pertinentia Trinitatis. (Quoniam in parte, dans A. FRIEDBERG, Corpus Iuris canonici editio Lipsiensis secunda post Aemilii Ludovici Richteri curas, t. 2 Decretaleum collectiones, Graz, 1959, Saint Grégoire, lib. II, tit. IX, De Feriis, chap. II.)

26 F. CABrol, Le culte de la Trinité dans la liturgie et l'institution de la fête de la Trinité, EPhemerides liturgicae, t. 45 (1931), pp. 270-278; P. Browe, Zur Geschichte des Dreifaltigkeitsfestes, ARCHIV Für LiturgIEWISSENSChaft, t. 1, 1950, pp. 65-81; Fl. Close, Le rôle de l'Église de Liège, pp. 112-115. 
la culture et de l'instruction dans le diocèse, le prestige et l'influence de l'école musicale liégeoise ainsi que le profond attachement des évêques mosans au culte divin et à la liturgie ${ }^{27}$.

\section{Une source d'inspiration indubitable: la Confessio de Trinitate d'Alcuin}

Richer fit acter sa décision d'ériger un oratoire doté d'un autel dédié à la Trinité dans la cathédrale Saint-Lambert. Il justifia son choix en réexposant l'essentiel de la doctrine chrétienne du monothéisme trinitaire en quelques formules bien senties dont l'ampleur correspond pratiquement à la moitié du texte copié par le chanoine Anselme (98 mots sur 206). L'insertion de ces extraits dans la charte de l'évêque de Liège mérite une attention particulière. Tous les passages à forte connotation théologique de ce document - tant ceux du protocole initial que du préambule - ont été littéralement puisés dans la Confessio de Trinitate - ou Symbolum fidei de sancta Trinitate - d'Alcuin, le diacre anglo-saxon conseiller de Charlemagne ${ }^{28}$. Cette profession de foi de structure binaire - Trinité et christologie - et de style rédactionnel ternaire cadencé, caractéristique des symboles de foi trinitaires de la basse antiquité, fut rédigée au tournant $\mathrm{du} \mathrm{Ix}^{\mathrm{e}}$ siècle. Dans la tradition manuscrite, elle se rattache à deux autres œuvres du diacre anglo-saxon: l'hymne Adesto et le traité De fide sanctae et individuae Trinitatis $^{29}$. Parfois présenté comme la première somme théologique médiévale, le $D e$ fide propose un exposé systématique de la doctrine trinitaire, une synthèse de ce que tout chrétien orthodoxe du haut Moyen Âge devait croire. Plus qu'une synthèse des connaissances religieuses de son temps, la rédaction de ce traité fut l'aboutissement triomphant du processus de clarification de la doctrine chrétienne, encouragé et nourri par les débats christologiques de la dernière décennie du viII $^{e}$ siècle. Sorte de lecture herméneutique du Credo à l'usage de l'élite intellectuelle de son temps, il s'imposa rapidement comme la référence en matière d'enseignement de la doctrine trinitaire. L'arrivée de ce traité à la cour impériale donna l'impulsion à une véritable campagne de promo-

27 Fl. Close, L'office de la Trinité. Liège, Metz, Mayence, Saint-Gall.

28 Alcuin, Confessio fidei, dans Patrologie Latine (ci-dessous $P L$ ), t. 101, Paris, 1863, col. 56-58.

29 Alcuin. De fide sanctae et individuae Trinitatis ad gloriosum imperatorem Carolum magnum Deo devotum Libri tres, dans PL, t. 101, Paris, 1863, col. 9-54; AlcuIN, Hymne Adesto, idem, col 54-56. Sur ces œuvres cf. M.-H. Jullien, Fr. Perelman (éd.), Clavis des auteurs latins du Moyen Âge. Territoire français, 735-987, t. 2 Alcuin, Turnhout, Brepols, 1999, ALC 28, pp. 134-139 et, en dernier lieu, J. CAVAdINI, The sources and theology of Alcuin's De fide sanctae et individuae Trinitatis, Traditio, t. 46, 1991, pp. 123-146. 
tion systématique de la doctrine trinitaire dans l'Occident carolingien ${ }^{30}$. Il n'est pas aisé d'apprécier dans quelle mesure le traité d'Alcuin put être mémorisé par les clercs. Par contre, l'impressionnante quantité de copies manuscrites de cette ouvre encore conservées de nos jours témoigne du formidable succès de ces trois œuvres - le De fide, l'hymne Adesto et la Confessio fidei - au haut Moyen Âge ${ }^{31}$. Le fait que l'évêque Richer usât d'une formule trinitaire empruntée à l'œuvre d'Alcuin n'est pas anodin. Il atteste que le rédacteur de cette charte avait parfaitement assimilé, voire mémorisé, la Confessio de Trinitate d'Alcuin. Il laisse supposer que ce textc s'était précédemment imposé comme la norme de définition de l'essence divine une et trine, comme le contenu essentiel de l'enseignement à dispenser universellement au peuple chrétien. Cette idée est corroborée par la formule retenue par Richer comme adresse de l'acte. La teneur de ce document résonne comme une véritable prédication de la doctrine trinitaire, fondement du monothéisme chrétien.

Au moment de composer son office liturgique de la Trinité, Étienne de Liège emprunta la majorité des textes qu'il mit en musique à l'hymne Adesto d'Alcuin ${ }^{32}$. Dans son remaniement de la Vita prima sancti Lamberti, Étienne n'hésita pas à placer dans la bouche de l'évêque martyr un exposé détaillé de la foi chrétienne qui évoque d'emblée les credo carolingiens à forte connotation trinitaire ${ }^{33}$. Près du tiers des mots qui composent la charte du 16 novembre 932 est extrait de la Confessio fidei du même auteur carolingien. Cet acte vient étayer la thèse de la continuité du programme de formation théologique du haut clergé de la réforme carolingienne, indépendamment du devenir politique et institutionnel de l'empire. En inscrivant la fondation de son autel dans la continuité de l'œuvre liturgique d'Étienne de Liège, Richer manifesta le souci commun aux évêques liégeois de poursuivre l'entreprise de promotion de la dévotion trinitaire amorcée plus d'un siècle auparavant.

${ }^{30}$ Ce traité fut offert à Charlemagne par son auteur au plus tard en mai 802 . W. HeIL (Der Adoptianismus, Alkuin und Spanien, dans B. Bischoff (éd.), Karl der Grosse, t. 2 Das geistige Leben, Düsseldorf, Schwann, pp. 147-148) a proposé la date de 801 . John Cavadini s'est rallié à l'hypothèse de Donald Bullough d'un don légèrement plus tardif (J. CAVADINI, The sources and theology of Alcuin's De fide, p. 125, n. 9). - Sur l'implication de Charlemagne dans la propagation de la doctrine trinitaire dans l'Église latine occidentale, voir Fl. Close, Prêcher la Trinité dans le royaume carolingien. Un facteur de cohésion de l'Imperium christianum?, Communication orale présentée aux Journées d'Études Prédication et Exégèse $\left({ }^{2} X^{e}-x I r^{2}\right)$ (Auxerre, 3-4 octobre 2008), dans UNIVERSITÉ DE LIÈGE, Open Repository and Bibliography, [en ligne] http://orbi.ulg.ac.be/handle/2268/11469; EAD, In nomine sanctae et individuae Trinitatis. Uniformiser la foi pour unifier le royaume franc, mémoire couronné par l'Académie Royale de Belgique (concours 2009), à paraître dans la collection des Mémoires de la Classe des lettres.

31 On compte à l'heure actuelle une centaine de manuscrits plus ou moins complets de cette œuvre connus et conservés dont vingt-huit du IX ${ }^{e}$ siècle (M.-H. Jullien, Fr. Perelman (éd.), Clavis, t. 2, ALC 28, pp. 134-139.)

32 Fl. Close, L'office de la Trinité d'Étienne de Liège (901-920).

33 ÉtIENNE DE LIÈGE, Vita secunda s. Lamberti, c. III, $\$$ 28-30, dans AASS, septembre, t. 5, Anvers, 1755 , pp. 586-587. 
Une confrontation de la copie historique de la charte de 932 éditée par R. Koepke et du symbole de foi d'Alcuin fait apparaître quelques modifications du texte, grammaticalement correctes mais lourdes de signification d'un point de vue théologique ${ }^{34}$. On pourrait arguer de la piètre qualité des éditions de la Patrologie latine pour douter de la version originale du texte d'Alcuin, si la leçon retenue par J.P. Migne ne se retrouvait dans les meilleurs manuscrits que nous avons eu l'occasion de consulter ${ }^{35}$. La plus remarquable de ces variantes de lecture est assurément l'omission - la suppression? - du verbe esse dans l'expression cui idem est esse vivere et intelligere. Sous la plume d'Alcuin, cette phrase présentait le DieuTrinité comme ayant trois puissances: l'être, le vivre et le comprendre. La formule estropiée de l'édition de R. Koepke fait perdre à cette expression théologique sa signification et son caractère ternaire ${ }^{36}$. De même, retenir la leçon cui proprium est quod sempiternum est, comme le fit $\mathrm{R}$. Koepke, plutôt que celle donnant le pronom relatif au datif (cui) revient à priver la Trinité de l'une de ses propriétés en rompant une nouvelle triade ${ }^{37}$. En admettant l'hypothèse de la mémorisation du symbole de foi d'Alcuin par l'évêque de Liège Richer, il nous paraît impossible que ces modifications textuelles qui, non seulement, portent atteinte à la définition doctrinale mais cassent le rythme de la formule, aient été l'œuvre d'un membre lettré du haut clergé du $\mathrm{x}^{\mathrm{e}}$ siècle. Forte des leçons de la tradition manuscrite, nous plaidons en faveur de la conformation de l'exposé théologique de Richer à la Confessio fidei d'Alcuin.

34 Une édition du traité sur la Trinité et des deux pièces qui lui sont traditionnellement annexées par J. Cavadini, E.A. Matter et C. Sheppard est annoncée chez Brepols depuis une bonne dizaine d'années (cf. M.H. Jullien, Fr. Perelman (éd.), Clavis, t. 2, p. 139). Dans l'attente de celle-ci, nous restons tributaires du texte de la Patrologie latine (cf. n. 29).

$35 \mathrm{Cf}$. annexe.

36 Cette triade être, vivre, comprendre est le schéma du modèle métaphysique, inspiré de la tradition néoplatonicienne que Marius Victorinus choisit pour penser le mystère trinitaire. Il développa largement cette définition de Dieu dans son traité Adversus arianum, IV, 18,12; 21, 26 - 22,6;26,10;28,10. (cf. MARIUs VICTORINus, Traités théologiques sur la Trinité, t. 1, éd. P. Henry, trad. P. Hadot, Paris, Cerf, 1960 (SC 68), introduction pp. 81-83). L'influence directe de ce rhéteur romain sur Alcuin est palpable. (W. GeERLINGs, art. Marius Victorinus, dans Lexikon für Theologie und Kirche, $3^{\mathrm{e}}$ édition, t. 6 (1997), col. 1387-1388; A. SoliGNAC, art. Marius Victorinus, dans Dictionnaire de Spiritualité Ascétique et Mystique, t. 10 (1980), col. 616-623.; P. HADOT, Marius Victorinus et Alcuin, ARCHIVES D'HISTOIRE DOCTRINALE ET LITTÉRAIRE DU MOYEN ÂGE, t. 21, a. 1954 (1955), pp. 5-19; ID., Les hymnes de Victorinus et les hymnes Adesto et Miserere d'Alcuin, idem, t. 27, a. 1960 (1961), pp. 7-16; J.A. JUNGMANN, Marius Victorinus in der karolingischen Gebetsliteratur und in römischen Dreifaltigskeitsoffizium, dans P. GRanfield, J. JungmanN, Kyriakon Festschrift Johannis Quasten, $2^{\mathbf{c}}$ éd., vol. I, Münster, Aschenhoff, 1970, pp. 691-697; J. CAvadINI, The sources and theology of Alcuin's De fide, p. 146).

37 Ce choix de l'éditeur est d'autant plus étonnant que trois des quatre manuscritsétalons de R. Koepke contenaient cette variante de lecture. (cf. $M G H S S$, t. 7, p. 200, apparat critique $\mathrm{m}$ ). 


\section{Datation de l'acte}

Richer date son acte en référence à la onzième année du règne d'Henri l'Oiseleur $^{38}$. Ce décompte des années de règne est surprenant. Henri fut élu roi de Germanie au mois de mai $919^{39}$ mais n'imposa sa souveraineté en Lotharingie que par étapes, entre 923 et 925 , au gré des querelles qui l'opposèrent successivement aux rois qui se succédèrent à cette époque en Francie occidentale. Selon la chronologie traditionnelle, l'année 932 ne correspond à la onzième année du règne d'Henri l'Oiseleur ni en Germanie, ni en Lotharingie. L'archevêque de Trèves et le comte Gislebert de Lotharingie retinrent l'année 923 comme point de départ du calcul du règne d'Henri en Lotharingie dans la datation de leurs chartes ${ }^{40}$. Si la «chancellerie » épiscopale liégeoise avait opté pour ce même terminus a quo, l'année 932 aurait correspondu non à la $X I^{e}$ mais à la $X^{e}$ année du règne d'Henri. Doit-on pour autant conclure que le rédacteur de l'acte s'est trompé ou qu'aucune des copies de la chronique d'Anselme ne nous a transmis fidèlement le contenu du document original? La réponse n'est peut-être pas aussi évidente. En effet, confirmé dans ses fonctions par le pape Jean $\mathrm{x}$ en novembre 921, Richer, abbé de la grande abbaye carolingienne de Prüm, fut élu évêque de Liège, au terme d'une âpre querelle de succession et ce, grâce à l'appui du dernier carolingien régnant, le roi de Francie occidentale, Charles le Simple. Cette élection se fit au détriment d'Hilduin, le candidat d'Henri $\mathrm{I}^{\mathrm{er}}$ et du comte de Lotharingie Gislebert, révolté contre Charles III ${ }^{41}$. Henri l'Oiseleur fut le premier «non-franc» élevé à la dignité royale dans le royaume carolingien de l'Est. Pour cette raison, il dut lutter durant les premières années de son règne contre les prétentions des grands. Dans de telles circonstances, il fut amené à renoncer provisoirement à ses prétentions sur la Lotharingie. Le 7 novembre 921, il rencontra Charles III sur une île rhénane non loin de Bonn et conclut avec lui une amicitia. Par ce traité dit «de Bonn», Charles fut reconnu roi de Lotharingie et Henri, roi des Francs ${ }^{42}$. Au même moment, Richer était confirmé dans sa mission épiscopale par le pape Jean x (4 novembre 921). Rentré à Liège, le nouvel

${ }^{38}$ Se référant à l'élection d'Henri en tant que roi de Francie orientale, au mois de mai 919 , R. Koepke compta quatorze années d'intervalle entre l'avènement d'Henri l'Oiseleur et l'établissement de ce document. (ANSELme, Gesta episcoporum, p. 201, n. 90). Influencé par ce décompte, il crut lire regnante Heinrico rege gloriosissimo anno $X V$ dans le manuscrit de Wolfenbüttel (Cod. Guelf. 76.14 Aug $2^{\circ}$, fol. $56 \mathrm{v}^{\circ}$ ). Au terme d'une minutieuse comparaison paléographique, nous osons affirmer que ce manuscrit comporte la mention anno $X I$ (cf. annexe).

39 E. KARPF, art. Heinrich I, dans Lexikon des Mittelalters (ci-dessous LMA), t. 4 (1989), col. 2036-2037.

40 C. BRÜHL, Naissance de deux peuples, Français et Allemands ( $I X^{e}-X t^{e}$ siècles), trad. de l'allemand par G. DuCHET-Suchaux, Paris, Fayard, 1994 [1990], pp. 197-202.

41 H. Zimmermann, Der Streit um das Lütticher Bistum vom Jahre 920-921, in MrTteiLUNGEN DES INSTITUTS FÜr ÖSTERREICHISCHE GESCHICHTSFORSCHUNG, t. 65 (1957), pp. 15-52.

42 K.F. Werner, art. Bonn, Vertrag, dans LMA, t. 2 (1983), col. 428-429; C. BrüHL, Naissance de deux peuples, pp. 198-200. 
évêque dut éprouver quelques difficultés à prendre possession de son siège; Gislebert reprit les hostilités contre Charles III dès l'hiver 921-922 ${ }^{43}$. Dans les mois qui suivirent, le pouvoir du dernier Carolingien, déjà bien ébranlé, chancelait; à la fin du mois de juin 922, le marquis Robert de Neustrie était couronné par les grands du royaume de Francie occidentale et sacré à Reims par l'archevêque de Sens. Le coup d'État des Robertiens et l'hostilité de Gislebert auraient-ils incité Richer, fidèle aux Carolingiens, à rallier le camp du roi de Germanie? Nous ne connaissons pas de document qui évoque précisément la situation politique dans le diocèse mosan durant cette période troublée. Par contre, on sait que Richer se montra fidèle à Henri $\mathrm{I}^{\mathrm{er}}$. Ne serait-il pas envisageable qu'il se soit rallié au roi de Germanie au lendemain de la chute du dernier Carolingien? La datation de la onzième année du règne d'Henri l'Oiseleur, davantage qu'une erreur de copiste, ne pourrait-elle être un indice isolé en faveur d'une anticipation de quelques mois de l'insertion du diocèse lotharingien dans la mouvance du territoire germanique?

\section{Conclusion}

Bien que les copies historiques soient généralement regardées avec méfiance par les diplomatistes, l'acte du 16 novembre 932 inséré dans le paragraphe 21 des Gesta episcoporum Tungrensium, Traiectensium et Leodiensium du chanoine Anselme mérite bien des égards. Tout d'abord, il est l'un des rares témoins de l'activité de la «chancellerie» épiscopale liégeoise de la première moitié $\mathrm{du} \mathrm{x}^{\mathrm{e}}$ siècle et, bien qu'incomplet, il s'impose comme un exemple intéressant des actes délivrés à l'époque; Anselme a veillé à reproduire scrupuleusement les différentes parties constitutives de l'acte. Ensuite, il nous apporte une information précieuse sur la persistance du programme de la réforme religieuse carolingienne en Lotharingie en dépit des bouleversements politiques et institutionnels qu'elle connut au fil des divisions successives de l'empire franc entre les descendants de Charlemagne. Plus particulièrement, l'acte de 932 pose la question de la présence à Liège d'un exemplaire de l'œuvre d'Alcuin ou, a contrario, celle de l'immixtion de la culture et de la spiritualité monastique dans les églises séculières de l'époque ${ }^{44}$. Enfin, il comporte peut-être dans sa formule

43 H. ZimmermanN, Der Streit um das Lütticher Bistum, pp. 32-33.

44 La dévotion particulière des moines bénédictins pour Dieu trine est attestée dès l'époque carolingienne, notamment par les nombreux monastères, églises et autels qui lui ont été dédiés. Le formulaire de la messe votive de la 'l'rinité qu'Alcuin envoya à l'extrême fin du VIII ${ }^{e}$ siècle aux communautés de Fulda et de Saint-Vaast a très rapidement trouvé sa place dans les missels de l'ordre de saint Benoît; au $\mathrm{IX}^{\mathrm{e}}$ siècle, l'idée du dimanche dédié à Dieu, Père, Fils et Esprit était acquise dans la liturgie monastique (P. BRowe, Zur Geschichte des Dreifaltigkeitsfestes, pp. 67-68; A. KLAus, Ursprung und Verbreitung der Dreifaltigkeitsmesse, Münster, 1938, pp. 76-107.) 
de datation une indication susceptible d'apporter quelques précisions à une page d'histoire liégeoise mal connue, celle de la prise de position des évêques de Liège en faveur de la Francia orientalis. Témoin de l'activité diplomatique, liturgique, culturelle et musicale liégeoise $\mathrm{du} \mathrm{x}^{\mathrm{e}}$ siècle, la charte du 16 novembre 932 mériterait, nous semble-t-il, sa place aux côtés des autres chartes délivrées par les évêques de Tongres-Maastricht-Liège.

G. Kurth, le premier, plaida en faveur de l'établissement d'une nouvelle édition critique de la chronique d'Anselme. L'étude d'un seul paragraphe de cette chronique a suffi pour nous convaincre de la pertinence de ces appels réitérés. Puissent-ils un jour être entendus ...

\section{ANNEXE}

Richer, évêque de Tongres-Liège rappelle que le formulaire liturgique de l'office de la Trinité a été composé par son prédécesseur l'évêque Étienne (901-920) et fonde un autel de la Trinité dans la cathédrale Saint-Lambert de Liège.

\section{2, 16 novembre}

A. Original perdu

Copies historiques insérees dans le paragraphe 21 des Gesta episcoporum Tungrensium, Traiectensium et Leodiensium d'Anselme de Liège :

Tradition A :

$A v$. COPIE HISTORIQUe MANUSCRITE du $\mathrm{XVI}^{\mathrm{e}}$ siècle faite sur un manuscrit qui pourrait être l'original dans Averbode, AA lezen Sectie IV, Nr. 12, p. 66-67.

L1. COPIE hISTORIQUE MANUSCRITE, datée de 1606 réalisée par Daniel Raymundi, chanoine de Saint-Materne de la cathédrale Saint-Lambert, d'après un manuscrit perdu du $\mathrm{XI}^{\mathrm{e}}$ ou du XII ${ }^{\mathrm{e}}$ siècle dans Liège, BU, ms 1964, fol. 91 v-92r.

Tradition B :

W. Copie historique manuscrite du XiI ${ }^{\mathrm{e}}$ siècle dans Wolfenbütel, Cod. Guelf. 76.14 Aug $2^{\circ}$, fol. $56 \mathrm{v}^{\circ 45}$

$P$. COPIE historique MANUSCRITE du XII ${ }^{\mathrm{e}}$ siècle provenant de l'abbaye d'Echternach dans Paris, BN, ms latin 11104, fol. 92r-92v.

$H$. Copie historique manusCrite du XII $^{\mathrm{e}}$ siècle dans Den Haag, KB, 72E9, fol. $41 \mathrm{v}-42 \mathrm{r}$.

L2. Copie historique manusCrite du XiII ${ }^{\mathrm{e}}$ siècle provenant de l'abbaye d'Aulne dans Liège, BU, ms 3173, fol. 45v-46r.

An. COPIE hISTORIQUE IMPRIMÉE du XVIII ${ }^{\mathrm{e}}$ siècle provenant de l'abbaye d'Andage (Saint-Hubcrt) dans: Gesta pontificum Trajectensium et Leodicnsium a s. Remaclo ad Vazonem. Ex MS codice Andaginensis monasterii S. Huberti ante

45 Fac simile en ligne: Herzog August Bibliothex Wolfenbüttel, Wolfenbütteler Digitale Bibliothek, http://diglib.hab.de/wdb.php"pointer=115\&dir=mss\%2F-76-14-aug-2f, (image 116). Copie sur parchemin XII siècle. $29 \times 20 \mathrm{~cm}$. Manuscrit sur parchemin réuni au $\mathrm{XVII}^{\mathrm{e}}$ siècle à un autre sur papier du Xve. Description du manuscrit dans Katalog der HerzogAugust - Bibliothek Wolfenbüttel. Die Alte Reihe Nachdruck der Ausgabe 1884-1913, t. 6 Die Augusteischen Handschriften 3, Francfort, Klostermann, 1966, p. 396-399, nº 2738. 
annos Sexcenios exarato, $\S 21$, éd. E. Martène et U. Durand, in Amplissima Collectio, t. 4, Paris, 1729, col. 858-859.

Éditions dans la chronique d'Anselme de Liège:

Libri Anselmi cum additis capitulis Aegidii a Leodio, Aureaevallis religiosi, c. 41, éd. J. ChaPEAVIlle, Qui gesta pontificum Tungrensium Traiectensium et Leodiensium scripserunt ..., t. 1, Liège, Typis Christiani, 1612, p. 170-171. (Édition de la version abrégée selon le Liège, BU ms. 178 ${ }^{46}$ )

ANSELME DE LIÈGE, Gesta episcoporum Tungrensium, Traiectensium et Leodiensium, $\S 21$, éd. R. KOEPKE, in MGH SS., t. 7, Hanovre, 1846, p. 200-201.

Anselme, Gesta episcoporum Leodiensium, éd. J.P. Migne, in Patrologie Latine, t. 139 (1880), col. 1084-1085 (selon éd. R. Koepke).

Analyse: A. Wauters, St. Bormans, J. Halkin et al., Table chronologique des chartes et diplômes imprimés concernant l'histoire de la Belgique, t. 1, Bruxelles, 1866, p. 342.

Exemplar testamenti in quo testatur Richarius $\left({ }^{a}\right)$ Stephanum condidisse responsoria de sancta $\left({ }^{b}\right)$ Trinitate

Omnibus sanctam Trinitatem confitentibus, Patrem et Filium et Spiritum sanctum $\left({ }^{c}\right)$, Patrem plenum Deum in se, Filium plenum Deum a Patre genitum, Spiritum sanctum plenum Deum a Patre et Filio procedentem, non tamen tres Deos sed unum Deum ${ }^{47}$, Richarius Tungrensis ecclesiae $\left({ }^{d}\right)$ episcopus ${ }^{48}$, gratia vobis et pax a Deo Patre et Filio eius domino nostro Jesu Christo et Spiritu sancto ${ }^{49}$. $\mathrm{Si}$ aliquid utilitatis vel fructificationis in ecclesiasticis $\left({ }^{e}\right)$ rebus nobis commissis ordinare valemus, quamtotius $(f)$ accelerare debemus. Idcirco noverit industria fidelium, tam praesentium $(g)$ quamque futurorum, quia $\left({ }^{h}\right)$ venerabilis vir, praecessor $\left({ }^{i}\right)$ videlicet noster Stephanus ${ }^{50}$, in honore sanctae $(j)$ Trinitatis quaedam $\left({ }^{k}\right)$ responsoria cum antiphonis nocturnalibus sive matutinalibus necnon vespertinalibus totumque ad plene officium dulcissimae $(l)$ modulationis stabilire curavit, credens sibi suacquc $\left({ }^{m}\right)$ ccclcsiac $\left({ }^{n}\right)$ liquido profuturum, si illi debitas laudes $\left({ }^{\circ}\right)$ speciatim deferret cui nichil addi $\left(^{(}\right)$vel minui potest, quia semper est quod est, cui proprium est, cui $\left({ }^{q}\right)$ sempiternum est, cui idem est $\left(^{(}\right)$esse $\left({ }^{s}\right)$ vivere et intelligere. Hanc quoque semitam vestigii suprascripti firmiter incedentes, curavimus quoddam oratorium in ecclesia $\left({ }^{t}\right)$ sancti Lamberti, ubi $\left({ }^{u}\right)$ ipse quiescit, struere $(v)$,

$\left({ }^{a}\right)$ Riharius testatur $L 2 .-\left({ }^{b}\right)$ S. Trinitate $A v .-\left({ }^{c}\right)$ Le scribe de $L 2$ a copié deux fois d'affilée les mots patrem plenum deum in se filium avant de barrer la première copie. $\left({ }^{d}\right)$ ecclesie $L 2$ - ęcclesię $W, P, H .-\left(^{(}\right)$ęcclesiasticis $W, P, H .-\left({ }^{(}\right)$quantocyus $L 1$. - quam tocius $P, H$. - quantocius $L 2, A n .-\left({ }^{g}\right)$ presentium $H-$ praesencium $L 2 .-\left({ }^{h}\right)$ quod $A n$. - (') praedecessor $L 1$. - ( ${ }^{(j)}$ sancte $W, H$ - sancte $L 2$. - $\left(^{k}\right)$ quedam $W, L 2$ - quędam $H$. (') dulcissimę $W$ - dulcissime $P, H, L 2 .-\left({ }^{(n)}\right)$ suęque $W, H-$ sueque $L 2 .-\left({ }^{n}\right)$ ęcclesię $W, P$, $H$ - ecclesie L2. - $\left(^{o}\right)$ laude $W .-\left({ }^{p}\right)$ cui addi nihil $H .-\left({ }^{q}\right)$ quod $A n .-\left({ }^{r}\right)$ Mot manquant dans $A n .-\left({ }^{s}\right)$ Mot manquant dans $W, P, H, L 2, A n$. - ( $\left.{ }^{(}\right)$ecclesia $W, P, H$. - (") quo $L 2$. (") Le scribe de $L 2$ a corrigé le texte en ajoutant con au-dessus de struere.

46 Cf. n. 13

47 Pater plenus Deus in se, Filius plenus Deus a Patre genitus, Spiritus sanctus plenus Deus a Patre et Filio procedens. Non tamen tres deos dicimus sed unum Deum omnipotentem (Alcuin, Confessio, PL 101, col. 57).

48 Richer, évêque de Liège (921/22 - 945).

49 Gratia et pax a Deo patre et Filio eius Jesu Christo Domino nostro sit ista confitenti in omnia saecula saeculorum (Alcuin, Confessio, col. 58C).

50 Étienne, évêque de Liège (901-920). 
atque in eo altare statuentes $\left({ }^{a}\right)$, quamvis a cunctis fidelibus Deus et Dominus vera et sempiterna unitas in substantia $\left({ }^{b}\right)$ ubique fideliter adoretur, quia $\left({ }^{c}\right)$ una est substantia $\left(^{(}\right)$Pater et Filius et Spiritus sanctus ${ }^{51}$, tamen in honore ipsius sanctae $\left({ }^{e}\right)$ Trinitatis dedicare maturavimus.

[Sequitur deinceps cuiusdam fundi ad idem altare traditio $(f)$ unde $(g)$ ibidem continentur $\left({ }^{h}\right)$ luminaria et reliqua divinae $\left({ }^{i}\right)$ servitutis iugiter adimpleantur $(j)$ solemnia $\left({ }^{k}\right)$ que $\left({ }^{l}\right)$ hic $\left({ }^{m}\right)$ interponere $\left({ }^{n}\right)$ supervacuum existimavi $\left.\left({ }^{o}\right)\right]^{52}$.

Actum est $\left({ }^{p}\right)$ hoc Leodii $\left({ }^{q}\right)$ sub die XVI. $\left({ }^{r}\right)$ Kalendas $\left({ }^{s}\right)$ decembris anno Domini $\left({ }^{\prime}\right)$ D.CCCC.XXX.II $\left({ }^{u}\right)$, indictione $\left({ }^{v}\right)$ V $\left({ }^{w}\right)$, regnante Heinrico rege ${ }^{53}$ gloriosissimo anno $\mathrm{XI}^{\mathrm{mo}}\left({ }^{x}\right)$.

$\left({ }^{a}\right)$ struentes $H, A n .-\left({ }^{b}\right)$ substancia $L 2 .-\left({ }^{c}\right)$ quod $A n$. - ( $\left.{ }^{(}\right)$substantia est $W, P, H$, $A n$ - substancia est $L 2 .-\left({ }^{e}\right)$ sanctę $W, H$ - sancte $L 2$. - $\left(^{f}\right)$ tradicio $L 2$. - $\left(^{g}\right)$ unde ut An. - $\left({ }^{h}\right)$ concinnentur $L 1-$ continetur $L 2 .-\left({ }^{i}\right)$ divinę $W, H-$ divine $L 2$. $-\left({ }^{(\jmath)}\right.$ adimple ntur $L 2 .-\left({ }^{k}\right)$ sollempnia $W, P, H, L 2 .-\left({ }^{\prime}\right)$ quae $L 1-$ quę $H .-\left({ }^{m}\right)$ in hic $W .-\left({ }^{n}\right)$ in tempore $W .-\left(^{\circ}\right)$ extimavi $W, P, H$ - aestimavi $A n .-\left(^{(p)}\right.$ Le scribe de $P$ a rajouté dans l'interligne supérieur le est qu'il avait manifestement oublié. $-\left({ }^{q}\right)$ Leodium $L 2 .-\left({ }^{r}\right) \mathrm{XVI}^{\circ}$ $L 1$ - decimo sexto $A n .-\left(^{s}\right) \mathrm{kl} W, L 2$. - ( $\left.{ }^{t}\right)$ Mot manquant $L 1 .-\left(^{u}\right)$ anno nongentesimo trigesimo secundo $L 1, A n-$ D.CCCC ${ }^{\text {nongentesimo }}$.XXX.II $W, H-$ D.CCCC ${ }^{\text {Nongentesimo }}$. $\mathrm{XXX}^{\mathrm{mo}}$.II $P-$ D.CCCC ${ }^{\text {Nongentesimo. }} \mathrm{XXX}^{\mathrm{mo}} . \mathrm{II}^{\circ} L 2 .-\left(^{\circ}\right)$ Inditione $W .-\left({ }^{\prime}\right)$ indictione quinta $L 1, A n-$ indictione $\mathrm{V}^{\mathrm{a}} W-$ indictione $\mathrm{V}^{\mathrm{ta}} P, H, L 2 .-\left(^{x}\right)$ anno undecimo $L 1, A n$ - anno XI $W$.

51 Cui nihil accidens esse poterit, quia simplici divinitatis naturae nil addi, vel minui potest, quia semper est quod est, cui proprium est, cui sempiternum est, cui idem est, esse, vivere, et intelligere. Et haec tria unus Deus, et unus Deus haec tria; idem Deus et Dominus vera sempiterna Trinitas in personis, vera et sempiterna Unitas in substantia, quia una est substantia Pater, et Filius, et Spiritus Sanctus. (Alcuin, Confessio, col. 57B).

52 Anselme a résumé en ces mots la suite du dispositif qui consistait, à ce qu'il semble, en une liste d'indications pratiques relatives aux luminaires et au service divin lors de la solennité.

${ }^{53}$ Henri ${ }^{\mathrm{er}}$ de Germanie dit l'Oiseleur (r. 919-936). 
RÉsuMÉ. - Le 16 novembre 932, l'évêque de Liège Richer consacra un autel à la sainte Trinité en la cathédrale Saint-Lambert de Liège et le dota richement; il consigna cette décision par écrit. La charte de fondation est parvenue jusqu'à nous sous la forme d'une copie historique isolée insérée dans les Gesta episcoporum Tungrensium et Leodiensium du chanoine Anselme de Liège. Cet article entend souligner l'intérêt de ce document tant pour l'histoire de la chancellerie liégeoise que pour celle de la réforme religieuse carolingienne dans l'espace lotharingien. Il comporte en annexe une nouvelle édition critique de ce document établie sur la base d'une confrontation de sept copies réparties en deux classes selon une typologic différente de celle de R. Koepke, l'éditeur de la chronique d'Anselme pour les Monumenta Germaniae Historica.

SAMENVATTING. - Op 16 november 932 wijdde de Luikse bisschop Richer een altaar aan de Heilige Drievuldigheid in de Sint-Lambertuskathedraal van Luik dat hij rijkelijk begiftigde. Hij stelde deze beslissing te boek. Het stichtingscharter is tot vandaag overgeleverd in de vorm van een geïsoleerde historische kopie die is opgenomen in de Gesta episcoporum Tungrensium et Leodiensium van de Luikse kanunnik Anselmus. In dit artikel wil de auteur het belang van dit document onderstrepen, zowel voor de geschiedenis van de Luikse kanselarij als voor de geschiedenis van de Karolingische religieuze hervorming in Lotharingen. Het artikel heeft als bijlage een nieuwe kritische editie van dit document, opgesteld op basis van een confrontatie van zeven kopieën die in twee klassen zijn verdeeld volgens een typologie die verschilt van die van $R$. Koepke, uitgever van de kroniek van Anselmus voor de Monumenta Germaniae Historica.

SUMmaRY. - On 16 November 932, Richer, bishop of Liège, devoted an altar to the Saint Trinity in Liège's St. Lambert's Cathedral and lavishly decorated it, a decision he recorded in writing. We obtained the foundation charter as an isolated historical copy, inserted into the Gesta episcoporum Tungrensium et Leodiensium from the Anselm of Liège canon. The article stresses the interest of this document as much for the history of the chancellery of Liège as for the Carolingian religious reform in the Lotharingian space. It has as an annex a new critical edition of the document, established based on a confrontation of seven copies divided into two classes according to a typology different from that of $\mathbf{R}$. Koepke, editor of the Anselm chronicle for the Monumenta Germaniae Historica. 